Résumés des conférences et travaux

\title{
Linguistique du breton
}

\section{Steve Hewitt}

\section{OpenEdition \\ Journals}

Édition électronique

URL : https://journals.openedition.org/ashp/404

DOI : 10.4000/ashp.404

ISSN : 1969-6310

Éditeur

Publications de l'École Pratique des Hautes Études

\section{Édition imprimée}

Date de publication : 1 octobre 2008

Pagination : 366-367

ISSN : 0766-0677

\section{Référence électronique}

Steve Hewitt, "Linguistique du breton », Annuaire de l'École pratique des hautes études (EPHE), Section des sciences historiques et philologiques [En ligne], 139 |2008, mis en ligne le 15 janvier 2009, consulté le 12 juillet 2021. URL : http://journals.openedition.org/ashp/404 ; DOI : https://doi.org/10.4000/ashp. 404 


\title{
LINGUISTIQUE DU BRETON
}

\author{
Chargé de conférences : M. Steve HewitT
}

Programme de l'année 2006-2007 : Les structures du breton moderne d'après le parler du Centre-Trégor-variations dialectales et diachroniques.

Cette année, nous avons continué l'examen des parlers vivants, surtout ceux de l'axe central NE-SO, en nous concentrant sur celui de Centre-Trégor (NE du domaine bretonnant). Nous avons d'ailleurs bénéficié de la présence de locuteurs natifs d'autres parlers (Léon [NO], SO Cornouaille, SE Cornouaille), ce qui a permis de fructueuses comparaisons. Nous avons étudié des documents sonores et écrits, en particulier concernant deux personnalités artistiques trégorroises, Fañch Danno et Maria Prat. Ce faisant, nous avons revu plusieurs phénomènes : les fricatives initiales (sourdes ou sonores) et les mutations consonantiques grammaticales correspondantes (souvent un son identique dans la chaîne parlée dans deux parlers différents a un statut différent vis-à-vis du système interne du parler en question - radicale dans un, consonne mutée dans un autre); les diverses formes et constructions du verbe « être » : eo (copule simple; syntaxe auxiliée), emañ (verbe situatif; syntaxe de verbe simple), so/eus (opérateur existentiel; syntaxe auxiliée), et la forme «d'habitude » veż qui se substitue aux trois formes précédentes au présent et à l'imparfait pour souligner le caractère habituel - cependant la syntaxe « auxiliée » ou « simple » de la forme « simple » est maintenue; et plus généralement, notre cadre d'analyse de la syntaxe verbale du breton : présentation « nue » avec syntagme prédicatif initial/présentation « introduite » avec un autre élément initial : sujet, objet, adverbe, etc., qui peut être soit thématique soit rhématique; syntaxe « verbe simple »/ « auxiliée »/ « dynamique/ double » avec un verbe lexical non-fini et un verbe grammatical fini séparés. Finalement, la morphologie du verbe « avoir » en breton m-eus [à.moi-il.y.a] «j'ai » fournit une clef pour expliquer le marquage scindé de la série de temps III «parfait, médiatif (évidentiel)» des verbes géorgiens, où les intransitifs inaccusatifs et les passifs ont un marquage « direct » (sujet au nominatif, marque du sujet dans le verbe et participe passé), mais les transitifs et les intransitifs inergatifs ont un marquage « indirect » (sujet au datif, marques d'objet indirect dans le verbe). Ainsi, ce marquage scindé en géorgien n'est pas idiosyncratique, comme pour la plupart des kartvélologues, mais se rattache bien sémantiquement et morphologiquement au système d'auxiliarité scindée de plusieurs langues européennes.

Géorgien

Classe 1 - verbes transitifs

$d a-m_{-} \quad i_{-} \quad x a t^{*} a v_{-} s$

PRF 1SG.OBL BEN peint SUF 3SG.DIR Je l'ai [apparemment] peint.
Breton

liwed $e$ m-eus aneżañ peint.PP AFF j'ai [1sG.OBL-être.EXIST] le.OBJ Je l'ai peint 
Classe 3 - verbes intransitifs inergatifs$$
m \text { - } i_{-} \quad m u \check{-}-a v_{-} n_{-} i_{-}
$$

1SG.OBL BEN travail SUF LC LV 3SG.DIR travaillé.PP AFF j'ai [1sG.oBL-être.EXIST] J'ai [apparemment] travaillé

J'ai travaillé

Classe 2 - verbes intransitifs inaccusatifs

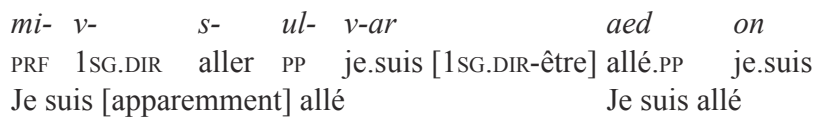

Classe 2 - verbes passifs
$d a-v-$
$x a t^{*}$ ul- v-ar
liwed
on (bed)
PRF 1SG.DIR peint PP I.am [1sG.DIR-être]
J'ai [apparemment] été peint
peint.pP je.suis (été.PP)
J'ai été peint 